\title{
Non-canonical Wnt/Ror2 signaling status regulates cell-matrix crosstalk to prompt directional tumor cell invasion and dissemination in breast cancer
}

Hongjiang $\mathrm{Si}^{1}$, Na Zhao ${ }^{1}$, Andrea Pedroza ${ }^{1}$, Chad Creighton ${ }^{2,3}$, Kevin Roarty ${ }^{1,2, \wedge, \#}$

${ }^{1}$ Department of Molecular and Cellular Biology, Baylor College of Medicine, Houston, TX 77030 ${ }^{2}$ Division of Biostatistics and Bioinformatics, Dan L. Duncan Comprehensive Cancer Center, Baylor College of Medicine, Houston, TX $77030{ }^{3}$ Department of Medicine, Baylor College of Medicine, Houston, TX 77030

^Corresponding author: Kevin Roarty roarty@bcm.edu

\#Twitter handle: @RoartyLab

\section{ABSTRACT}

Cancer deaths largely result from metastasis, the spread of cancer from the primary tumor to distant organs. Initial steps of metastasis require that tumor cells invade into the surrounding tissue and gain access to blood or lymphatic vessels. Such invasion is reliant on a balance of cell-cell and cell-matrix cues within the microenvironment of the tumor, yet factors regulating such interactions for invading tumor cells remain elusive in the context of cancer. We demonstrate that the noncanonical Wnt receptor, Ror2, in mammary tumor models of Tripe Negative Breast Cancer, regulates the composition and remodeling of the tumor stroma, where Ror2-depletion prompts directional tumor cell invasion and coordinated ECM production at the leading edge of tumor cell movement. By RNA sequencing, we discovered that tumor organoids specifically harbor actin cytoskeleton, cell adhesion, 
and collagen cross-linking gene expression programs when Wnt/Ror2 signaling is impaired. Interestingly, Ror2 depletion resulted in the downregulation of E-cadherin in tumor organoids, particularly at invading tumor cell protrusions within the surrounding ECM. Spatially, we identified the upregulation and redistribution of integrin receptors, particularly integrin- $\alpha 5$ in Ror2-deficient tumor organoids, accompanied by the simultaneous production of a provisional Fibronectin matrix, a requisite component of the ECM, ligand for integrin $\alpha 5$, and mediator of collagen assembly and organization. Along with altered ECM architecture, Ror2 loss reshaped the topology of integrin and FAK activation within primary tumors, suggesting an important physiological function for Ror2 in shaping both signaling and ECM architecture during tumor progression. Blocking either integrin or FAK, a downstream mediator of integrin-mediated signal transduction, abrogated the enhanced migration observed upon Ror2 loss. These results suggest that Ror2 status within a tumor can significantly impact adhesive vs. migratory states in breast cancer and provide a novel mechanism where Wnt/Ror2 shapes not only tumor cell composition, but also reciprocal cell-ECM interactions prompting directional and collaborative tumor cell transit during cancer progression.

\section{INTRODUCTION}

Metastasis, a multistep process where cancer cells leave the primary tumor to establish residence in a secondary organ site, is the primary culprit of cancer-related deaths. Distinct from molecular programs guiding transformation or proliferation of cancer cells, mechanisms regulating the dissemination and spread of tumor cells to distant sites within the body are distinct and likely evolve in a spatiotemporal manner dependent on the stage of metastasis. The initial steps of cancer metastasis require that tumor cells actively invade and disseminate from the originating primary tumor site by breaching the basement membrane and acquiring access to vasculature in order to disseminate to secondary organs ${ }^{1}$. Such a process requires that tumor cells activate signaling pathways that enable remodeling of the actin cytoskeleton while simultaneously tuning cell-cell and cell-matrix adhesions during invasion and cellular transit within the surrounding stroma ${ }^{2}$. Despite 
extensive investigation and categorization of mechanisms of cell migration governing both developmental and cancer contexts, such attempts are confounded by the inherent cellular complexity and heterogeneity of a tumor ${ }^{3}$. Several conserved mechanisms and cell machineries are activated as cells migrate in both normal and cancerous tissues, yet both cellular and microenvironmental heterogeneity in cancer, together with variations in migration strategies, have hindered recent efforts to thwart the initial invasion and dissemination stages responsible for spurring cancer metastasis.

Invasion of tumor cells into the stroma is accompanied by the reorganization of the surrounding extracellular matrix to enable a molecular scaffold conducive for tumor cell transit. In breast cancer, fibrosis and ECM stiffening is associated with poor prognosis in patients ${ }^{4-7}$. Elevated stiffening of the tumor ECM can occur through increased deposition, remodeling, and cross-linking of fibrillar type I collagen. Moreover, other matrix proteins like fibronectin can be assembled with type I collagen and enable lysyl oxidase (LOX)- or LOX-like dependent collagen crosslinking and organization $^{8}$. Tumor cells respond to altered ECM composition primarily through integrin receptors, the physical link between the actin cytoskeleton within the cell and the outside ECM. Such cell-ECM interactions prompt FAK autophosphorylation within the cell to reorganize its actin cytoskeleton, promote the assembly/disassembly of focal adhesions complexes, and apply traction forces to ECM proteins to facilitate directional cellular migration ${ }^{9}$. Integrins heterodimerize, allowing for a range of specificities to various ECM ligands, and such ligand-receptor interactions permit cancer cell survival, dissemination, transit, and colonization of tumor cells during metastatic progression ${ }^{10}$.

A growing body of evidence now suggests that cancer cell invasion takes on a variety of forms and likely involves the integration of individual and collective cell behaviors ${ }^{11}$. Accordingly, similar to morphogenic and patterning processes in development, cancer cells undergo dynamic fluctuations in cell polarity, cytoskeletal organization, and cell-cell cohesion during cellular movement, highlighting the importance of tumor cell coordination and reciprocal engagement with their surrounding 
microenvironment ${ }^{12,13}$. The precise regulation of such cellular behaviors and the signals that trigger invasive cell behavior in the context of cancer progression remain unknown.

Wnt signaling is a known regulator of cell fate, migration, and polarity during key morphogenic events in embryonic and postnatal development. Often such patterning requires simultaneous cell fate specification concomitantly with spatial positioning of the cells in order to achieve coordinated morphogenesis and dynamic cell movements ${ }^{14}$. While canonical Wnt/ $\beta$-catenin-dependent signals stabilize intracellular $\beta$-catenin and dictate self-renewal and cell-fate choices, alternative Wnt/ $\beta$ catenin-independent signals coordinate various processes associated with cell movements, such as planar cell polarity and convergent extension ${ }^{15}$. Much like patterned organisms and tissues, tumors comprise a hierarchy of cell types with a range molecular and phenotypic heterogeneity ${ }^{16}$. Such cellular diversity is often complicated by the presence of spatial and temporal fluctuations throughout tumor progression, highlighting the need to further dissect the spatiotemporal behavior of tumor cells during cancer progression and the consequences of such fluctuations.

We previously discovered that the intratumor landscape within basal-like Triple Negative Breast Cancer (TNBC) harbored tumor cells that differentially utilized distinct modes of Wnt signaling, demarcated by canonical Wnt/ß-catenin-active cells co-existing with tumor cells expressing Ror2, an alternative $\beta$-catenin-independent Wnt receptor ${ }^{17}$. Genetic depletion of Ror2 in primary tumors yielded increased stromal remodeling and collagen deposition, a poor prognostic factor for breast cancer patients ${ }^{18-20}$. Using a 3-dimensional (3D) Type 1 collagen model of tumor organoids, we identified gene expression programs highly representative of cell adhesion, collagen fibril organization, and cell-ECM alterations as a consequence of Ror2 loss. In particular, junctional E-cadherin expression was compromised upon Ror2 depletion, accompanied by an increase in tumor cell invasive into the surrounding collagen matrix. We uncovered the upregulation of integrin-mediated signaling in Ror2deficient tumor cells, particularly the expression $\alpha_{5}$ integrin and $\beta_{3}$ integrin at the invasive front of tumor organoids. Moreover, fibronectin (FN) was coincidentally upregulated at sites of invasion upon Ror2-loss. Such changes in integrin receptor abundance and localization additionally triggered 
changes in the cytoskeletal architecture of Ror2-deficient tumor cells, prompting an increase in $F / G$ actin ratios and the activation of focal-adhesion kinase (FAK). Inhibition of either integrin or FAK activation abrogated the heightened invasion prompted by Ror2-loss. These findings therefore reveal a critical function for the alternative Wnt receptor, Ror2, as a determinant of bi-directional communication between tumor cells and the ECM during cancer invasion and metastasis.

\section{RESULTS}

\section{Ror2 presence dictates tumor cell-directed collagen remodeling and transit through the ECM}

We previously discovered the coexistence of spatially distinct canonical $\mathrm{Wnt} / \beta$-catenin active and Ror2-expressing $\beta$-catenin-independent tumor subpopulations within the cellular landscape of TP53null TNBC basal-like models of breast cancer ${ }^{17}$. In experimental models, while Ror2 status impacted the proportion of molecularly distinct basal-like (BL) $\mathrm{CD}_{2} 4^{+} \mathrm{CD} 29^{+}$and claudin-low (CL) CD24 ${ }^{\text {llow }} \mathrm{CD}_{29^{+}}$within tumors, we also discovered a reorganization of the tumor stroma upon Ror2 depletion and further probed the functional implications of this altered stroma. Trichrome staining of Ror2-depleted tumors revealed a significant increase in collagen abundance and disorganized tumor architecture relative to Ror2-intact tumors (Figure 1A,B). We conducted 3D-cultures of tumor organoids within embedded collagen ECM to evaluate the functional impact of Ror2-depletion on adhesion and invasion, given that Collagen I is the most abundant scaffolding protein present in tissues and its crosslinking is highly associated with breast cancer risk. Using two independent Genetically Engineered Mouse (GEM) TP53-null transplant models ${ }^{17,21}$ representative of the basallike subtype of breast cancer (2225L and $2153 \mathrm{~L}$ ), we generated tumor organoids to probe the functional implication of lentiviral shRNA-depletion of Ror2 in a predominantly collagen-rich ECM. Ror2 loss prompted the active invasion of tumor cells into the surrounding collagen-rich ECM, accompanied by a 4.5 -fold and 3.7 -fold increase in invasive nodes within 2225L- and 2153L-shRor2 organoids, respectively, compared to control shLUC organoids (Figure 1C-D, E). 
We further investigated defects in epithelial and mesenchymal states given our previous observations that Ror2 loss prompted an enriched CL subpopulation and molecular signature in tumors $^{17}$. Such states can dictate both single and collective behaviors of tumor cells during invasion and dissemination, so we reasoned that aspects of EMT could be involved in Ror2 loss-of-function. Importantly, while E-cadherin was consistently downregulated upon Ror2 depletion (Figure 1H), the mesenchymal marker Vimentin was equally expressed in Ror2-intact vs. -deleted organoids (Figure 1I). Interestingly, junctional E-cadherin was moderately disrupted at the cell cortex in the body of shRor2 organoids, yet consistently decreased to a greater extent as cells invaded into the surrounding extracellular matrix (Figure1F-G'). While vimentin expression was not altered across organoids, the distribution of vimentin localization occasionally shifted such that invasive nodes in Ror2-depleted organoids harbored vimentin at protrusive areas of ECM involvement where Ecadherin expression was most diminished (Figure1F-G'). No notable change in other mesenchymalassociated transcription factors such as Zeb1, Snai1, and Snai2 were observed upon Ror2 loss (Figure 1J). The disruption in E-cadherin expression and lack of change in mesenchymal markers suggest that cell-cell adhesions are compromised, but not lost, upon Ror2 depletion rather than a complete shift from an epithelial to mesenchymal state.

\section{Ror2 depletion prompts alterations in actin cytoskeleton and cell-ECM dynamics}

The enhanced degree of invasion in tumor organoids along with increased collagen presence within Ror2-depleted tumors prompted us to further define specific gene expression programs directly impacted downstream of Ror2 loss that might contribute to the invasive switch. We performed RNA sequencing on 4-day 3D-cultured shLUC vs. shRor2 tumor organoids in Collagen I derived from basal-like 2225L and 2153L TP53-null models (Figure 2A-D). Evaluation of differentially expressed genes in response to Ror2 depletion revealed the representation of gene ontology terms encompassing the guidance of tumor cell movement, particularly the regulation of cell migration, cellcell adhesion, integrin-mediated adhesion, collagen fibril organization, and cytoskeletal organization 
(Figure 2B,D). Given the increased formation of collagen within tumor landscape upon Ror2 loss, we were particularly interested in how Ror2 presence shapes states of intercellular cohesion and appropriate exchanges between tumor cells and the ECM. Interestingly, LOX and LOX-like 2 (LoxI2), enzymes required for the biogenesis and crosslinking of fibrillar collagen, were upregulated upon Ror2 loss (Figure 2E,F). Additionally, $\alpha_{5}$ integrin and $\beta_{3}$ integrin, receptors for ECM components responsible for physically bridging internal and external filamentous networks and enabling bidirectional transmission of signals across the plasma membrane, were also elevated upon Ror2 loss (Figure 2E,F). Expression of $\alpha$ - and $\gamma$-actin molecules were also elevated, while cofilin, an actin depolymerizing factor, was reduced upon Ror2 depletion (Figure 2E,F). From these data, we postulated that Wnt/Ror2 signaling regulates an integral signaling juncture for the cell by shaping integrin presence and distribution, cytoskeletal adjustments, and tumor cell exchanges with the ECM.

\section{Loss of Ror2 triggers the upregulation and redistribution of integrins to assemble fibronectin}

\section{at sites of invasion}

Integrins are transmembrane receptors that mediate cell adhesion, migration, and enable the bidirectional signal transduction between cells and ECM proteins ${ }^{22}$. Given the strong presence of collagen in vivo within Ror2-deficient tumors, as well as the induction of collagen cross-linking enzymes Lox and Loxl2 in organoid cultures upon Ror2 downregulation (Figure 2E,F), we wanted to follow up on RNA sequencing identification of integrin changes within shRor2 organoids given their involvement in both the biogenesis of and interaction with ECM proteins during several cell migration processes $^{23}$. In shRor2 organoids, we verified that $\alpha_{5}$ integrin was upregulated at both the RNA and protein level relative to control shLUC organoids with intact Ror2 expression (Figure 2F, 3A). Interestingly, immunofluorescence staining for $\alpha_{5}$ integrin in tumor organoids revealed the upregulation and spatial localization of $\alpha_{5}$ integrin specifically at sites of tumor cell invasion into the surrounding Type I collagen matrix in Ror2-deficient organoids (Figure B,C). These data suggest a 
localized and directional shift for tumor cells to invade from the organoid body into the surrounding ECM, likely initiated by the disruption in junctional E-cadherin within Ror2-depleted organoids.

Integrins are $\alpha / \beta$ heterodimeric transmembrane receptors, and when the $\alpha_{5}$ subunit of integrin associates with $\beta_{1}$ integrin, this complex forms a fibronectin receptor ${ }^{23,24}$. Although 3D cultures were established in Type I collagen, we were particularly interested in probing tumor-cell derived fibronectin production as a consequence of Ror2 depletion since 1) integrins play a central role in fibronectin matrix assembly in addition to their role in cell adhesion, invasion, and bidirectional signaling, and 2) the deposition and restructuring of collagen in the ECM depends on the presence and stability of fibronectin. Indeed, fibronectin levels were elevated in shRor2 compared with shLUC organoids, despite an absence of this trend at the RNA level from the RNA sequencing data (Figure 3F). Moreover, like $\alpha_{5}$ integrin, fibronectin was spatially positioned adjacent to the invading cells disseminating from the organoid body upon Ror2 loss (Figure $\mathbf{3 G}, \mathbf{H}$ ). Intriguingly, the in vivo intratumoral topology of both $\alpha_{5}$ integrin and fibronectin shifted from the tumor periphery to throughout the tumor upon Ror2 loss (Figure 3D-E, 3I-J). Collectively, these data suggest the tumor-derived fibronectin helps facilitate integrin-mediated invasion into the adjacent cellular matrix, as has been demonstrated for directional migration maintenance in prostate cancer cells ${ }^{25}$ and fibroblasts ${ }^{26,27}$, as well as developmental processes encompassing convergent extension and oriented cell division ${ }^{28,29}$.

\section{Focal adhesions and actin cytoskeleton dynamics are regulated by Ror2}

Integrin-linked focal adhesion complexes serve as a physical scaffold for the cell to adhere to the exterior ECM and additionally interact with the interior actin cytoskeleton, providing instructive and highly tunable cues necessary for modulating adhesion and passage through the $\mathrm{ECM}^{9}$. Focal adhesion kinase (FAK) is activated downstream of integrins, typically in response to integrin clustering. Given the upregulation and localization of $\alpha_{5}$ integrin at plasma membrane sights of invasion in Ror2-deficient tumor organoids, we assessed the activation of FAK in both organoids and tumors with either intact- or deficient-Ror2 status. Interesting, the level of p-FAK was upregulated in 
tumor cells upon Ror2 loss in organoid cultures by western blotting (Figure 4A). This observation was consistent within 3D-cultured organoids when assessing p-FAK immunofluorescence (Figure 4B,C). When probing the intratumoral landscape of $\mathrm{p}-\mathrm{FAK}$ in vivo, we observed the prevalence of $\mathrm{p}$ FAK within tumor cells at the tumor periphery, while p-FAK levels were practically undetectable within the body of the tumor (Figure 4D). Ror2-depleted tumors had an overall increase in p-FAK levels within tumor cells of the periphery and central body of the tumor, suggesting Ror2 loss and consequently the altered ECM landscape in vivo, expanded the tumor topology of FAK activation from the periphery to throughout the tumor (Figure 4E).

As a consequence of altered p-FAK activation, we speculated that actin cytoskeletal dynamics were influenced by Ror2 presence. Indeed, cytoskeletal rearrangements were prompted by the Factin/G-actin turnover, as Ror2-depleted tumor cells exhibited an almost 4-fold higher level of filamentous $(F)$ to monomeric $(G)$ actin (Figure $\mathbf{4 F , G}$ ). Such findings suggest that Ror2 normally maintains some degree of actin cytoskeletal homeostasis within tumor cells by maintaining appropriate cell-cell and cell-ECM cues. In line with these findings, cofilin, a key actin-binding protein responsible for the depolymerization of filamentous $\mathrm{F}$-actin ${ }^{30}$, was downregulated in tumor organoids upon Ror2 loss (Figure 4H-J). We additionally detected an increase in the extent of p-Cofilin at Ser3 in the periphery of shRor2 tumors relative to a very narrow zone directly abutting the tumor-stromal interface in shLUC control tumors (Figure $4 \mathrm{~K}, \mathrm{~L}$ ) . Such a phosphorylation event inactivates cofilin, suggesting that heightened p-cofilin levels following Ror2 loss promote actin filament elongation, an increase in $F / G$ actin ratio, and consequently invasive potential of tumor cells. Lastly, phosphorylated Ezrin, Radixin, and Moesin (pERM), linkers of the actin cytoskeleton to the plasma membrane, were activated at the tumor periphery of control shLUC tumors but were expanded spatially throughout the tumor body upon Ror2 loss (Figure 4M,N). Moreover, while pERM was located at the cell cortex of shLUC tumor cells, a discontinuous punctate localization of pERM was prevalent in shRor2 tumor cells, likely reflecting altered intercellular and cell-ECM cues. 


\section{Inhibition of integrin and FAK impedes directional migration prompted by Ror2 loss}

To test the implications of integrin and FAK activation on Ror2 loss-of-function phenotypes with respect to invasion and dissemination, we tested small molecular inhibitors of both integrin and FAK activation. ATN-16, a novel small molecule peptide antagonist of integrin $\alpha_{5} \beta 1$, succesully inhibited pFAK activation prompted by Ror2 loss in a dose-dependent manner (Figure 5A). We subjected 2225L TP53null 2D cells to a wound healing assay to test the outcome of ATN-16 on the ability of shRor2 cells to efficient close the wound in culture. After 16 hours, shRor2 cells were more efficient at filling the area of wounding than control shLUC cells; however, $10 \mu \mathrm{M}$ administration of ATN-161 significantly impaired Ror2-deficient cells from migrating and closing the wound to a greater extent than shLUC control cells (Figure 5B-D). The inhibitor PF-562271 Besylate, a potent ATP-competitive reversible inhibitor of FAK activation, was also tested given the elevated $\mathrm{p}$-FAK levels in both organoids and tumors upon Ror2 loss. Treatment with besylate successfully mitigated the activation of FAK phosphorylation upon Ror2 loss (Figure 5E). Moreover, migration of shRor2 tumor cells was greatly hindered upon besylate treatment in 2D would healing assays (Figure 5F,G). Such a reduction in migration and invasion was additionally observed in 3D organoid cultures, where the cellECM landscape is quite different (Figure $\mathbf{5 H}-\mathbf{J})$. Thus, these studies place integrin engagement and FAK activation downstream of Ror2-loss and provide a mechanism to hinder the enhanced invasion and dissemination of tumor cells across various spatiotemporal contexts during breast cancer progression, where Wnt/Ror2 signaling is transiently and/or permanently impaired.

\section{DISCUSSION}

Cancer cells exhibit extremely adaptable cellular programs enabling their successful dissemination, survival, transit, and establishment of distant metastases. Such proficiency in migration and invasion mechanisms requires extensive cell-ECM exchanges and signaling events which guide and mobilize tumor cells. We demonstrate that the noncanonical Wnt receptor, Ror2, regulates intercellular adhesion and cell-ECM interactions impacting directional tumor cell invasion and coordinated ECM 
production to facilitate cancer cell invasion. By RNA sequencing, we uncovered that compromised Wnt/Ror2 signaling in vivo and within 3D-cultured tumor organoids specifically disrupts actin dynamics, adhesion, and collagen cross-linking gene expression programs likely conducive for tumor cell transit from the primary tumor. Interestingly, E-cadherin downregulation was observed upon Ror2 loss, particularly at invading tumor cell protrusions within the surrounding ECM (Figure 1). Integrin receptors, specifically integrin- $\alpha 5$ and integrin- $\beta 3$, were upregulated and spatially repositioned to the invasive front and cell-ECM interface of Ror2-deficient tumor organoids (Figure2,3). This change in integrin presence and clustering was also accompanied by the simultaneous production of a provisional Fibronectin matrix, a vital component of the ECM, ligand for integrin $\alpha 5$, and mediator of collagen assembly and organization ${ }^{31}$. Along with altered ECM architecture, Ror2 loss changed the intratumor spatial landscape of integrin and FAK activation within primary tumors (Figure 3,4), suggesting an important physiological function for Ror2 in shaping both signaling and ECM architecture during tumor progression. Blocking either integrin or FAK, a downstream mediator of integrin-mediated signal transduction, inhibited the migration and dissemination observed upon Ror2 depletion (Figure 5).

The cellular and molecular mechanisms involving tumor cell spread remain obscure. EMT has gained considerable attention as a mediator of cancer cell migration, particularly through the binary loss of adherens junction components (E-cadherin) and tight junctions (claudins) and upregulation of mesenchymal transcription factors Snail, Twist, and Zeb1; however, emerging evidence now demonstrates that collective cell migration likely also contributes to invasion and metastasis in cancer $^{32}$. Nonetheless, the interactions between the tumor ECM and cytoskeleton of the cancer cell are fundamental for defining the invasive potential of cancer cells. In the current study, we established that depletion of Ror2 levels in tumor cells disrupts the levels of E-cadherin and its localization within tumor cell junctions. E-cadherin loss was most pronounced in tumor cells that were observed migrating into the surrounding ECM. Although we could occasionally observe the concomitant expression of the mesenchymal marker, Vimentin, in invasive areas with E-cadherin loss 
(Figure 1), we did not observe the overt upregulation of mesenchymal genes in shRor2 organoids, suggesting the downregulation of junctional E-cadherin predominantly occurred in the absence of a distinct mesenchymal switch after Ror2 downregulation. Such observations therefore suggest a compromised state of cell-cell adhesion and altered intercellular coordination, enabling tumor cells to invade into the adjacent stroma. The regulation of junctional mechanocoupling by Wnt5a/Ror2 has been observed during angiogenic collective migration, where Wnt5a activated Cdc42 at cell junctions and stabilized vinculin/ $\alpha$-catenin binding to support adherens junction coupling with the actin cytoskeleton ${ }^{33}$. Interestingly, vinculin levels were upregulated as a consequence of Ror2 deletion in our models, suggesting potential compensatory mechanisms in response to E-cadherin loss and altered mechanoregulation of cell-cell adhesion in tumor cells ${ }^{34}$.

The alteration in tumor stroma, particularly the increased presence of Type I collagen upon Wnt/Ror2 disruption (Figure 1A), prompted us to probe Ror2's function in controlling cell-cell and cellmatrix interactions in tumor progression. In breast and other cancers, ECM stiffness results in poor patient prognosis, and alterations in collagen homeostasis, particularly the crosslinking and remodeling of collagen, can significantly impact tumor cell invasion and metastasis ${ }^{35}$. In multiple basal-like TP53null GEM models of breast cancer, we identified heightened representation of gene ontology terms which captured changes in collagen-crosslinking and cell-ECM function together with the actin cytoskeleton and cell adhesion. We discovered that Ror2 presence dictated the abundance and activation of key proteins integral for tuning cell-ECM exchanges. The heightened integrin expression upon Ror2 loss, particularly $\alpha 5$ integrin, prompted us to look further into the composition and reinforcement of ECM cues that enable Ror2-deficient tumor cells to migrate and invade. Fibronectin is a known structural ECM protein which binds $\alpha 5$ integrin, and its polymerization is known to regulate the composition and stability of extracellular matrix fibrils, particularly type I collagen $^{36,37}$. Intriguingly, we not only detected the clustering and upregulation of $\alpha 5$ integrin at sites of tumor cell invasion, but we also detected the deposition of fibronectin specifically at the interface of Ror2-depleted tumor cells with the surrounding ECM (Figure 3). These data, together with other 
studies that demonstrate fibronectin can promote directional persistence of cancer cell invasion ${ }^{38}$, suggest that alternative Wnt/Ror2 signaling can regulate invasion by modulating ECM composition along with adhesion properties of the cell. Notably, Wnt/PCP signaling through Wnt/Glipican4/Frizzled noncanonical Wnt signaling have been shown to regulate ECM assembly through effects on cadherin-mediated cell cohesion and that VANGL/Prickl1a actually have opposing functions on ECM organization with respect to fibronectin assembly in the zebrafish embryo ${ }^{28}$. Other studies have implicated Wnt/PCP as an essential mediator of integrin transmission of cytoskeletal tension required to direct fibronectin fibril formation at cell surfaces during convergent extension in Xenopus embryos ${ }^{39}$. As in vertebrate development, our study suggests alternative Wnt signaling through Ror2 plays an important role in mediating cell-matrix interactions that shape contextdependent spatial and temporal steps of metastasis. Given our previous findings that canonical and noncanonical Wnt signaling modes are integrated within mammary development and breast cancer settings ${ }^{17,40}$, further characterizing Wnt pathway coordination during tumor progression and metastasis will be necessary going forward. Defining such signaling pathways that shape adhesive and migratory states of a tumor will provide a more comprehensive understanding of the phenotypic variation of tumor cells and the spatiotemporal states of signaling guiding both the composition and cooperativity of cell-ECM entities during cancer metastasis.

\section{Materials and methods}

\section{Mouse strains and mammary tumor maintenance}

This study is compliant with the rules of the Guide for the Care and Use of Laboratory Animals of the National Institutes of Health. The animal maintenance and procedures are approved by the Baylor College of Medicine Institutional Animal Care and Use Committee (Protocol AN-504). A transplantable TP53-null mammary tumor bank was generated as described ${ }^{21}$. The subtype $2225 \mathrm{~L}$ was maintained and propagated in the Balb/c inbred female mice (strain \#047, 3-4 weeks of age) purchased from ENVIGO (Houston, TX, USA). 
To generate shLUC and shRor2 2225L tumors, primary 2225L tumor cells were isolated and transduced as described below. Cells were then injected into cleared mammary fat pads at 25,000 cells $/ 10 \square \mu$ injection volume. Mammary tumors were allowed to grow until diameter reaches $0.5-1 \mathrm{~cm}$ before collection or propagation.

\section{Tumor cell isolation and culture}

Mammary tumors were collected at a diameter of approximately $1 \mathrm{~cm}$. Tumor tissues were minced into pieces $1 \times 1 \mathrm{~mm}$ and dissociated in HBSS containing $1 \mathrm{mg} / \mathrm{ml}$ collagenase A (\#11088793001; Roche, Basel, Switzerland) and $1 \mu \mathrm{g} / \mathrm{ml}$ DNase I (\#07900; Stemcell Technologies, Vancouver, Canada) in a shaking incubator at $125 \mathrm{rpm}$. and $37 \square^{\circ} \mathrm{C}$ for 2 hours. Tissues were pipetted and mixed every 20 minutes for homogeneous digestion. To enrich for tumor organoids, digests were centrifuged 4 times at $1500 \mathrm{rpm}$. for 8 seconds. Enriched organoids were subjected to $0.05 \%$ trypsin at $37 \square^{\circ} \mathrm{C}$ for $5 \square \mathrm{min}$, washed and filtered through a $0.40-\mu \mathrm{m}$ cell strainer to obtain single cells. Single cells were washed and used in further experiments.

In 2D culture, single cells were cultured in DMEM/F-12 medium (\#11320033; ThermoFisher Scientific, Waltham, MA, USA) supplied with 10\% FBS (\#10082147; ThermoFisher Scientific), $5 \mu \mathrm{g} / \mathrm{ml}$ insulin (\#I5500; Sigma-Aldrich, St. Louis, MO, USA), 1 gg/ml hydrocortisone (\#H0888; Sigma-Aldrich), and 10 ng/ml mEGF (\#SRP3196, Sigma-Aldrich). $200 \mu \mathrm{g} / \mathrm{ml}$ Geneticin (\#10131035; ThermoFisher Scientific) was added to select for tumor cells. In organoids culture, single cells (500,000 cells/well) were seeded in Ultra-low attachment 24-well plates (\#3473, Corning, Corning, NY, USA) for overnight aggregation. Aggregated organoids were further used in tumor organoids assays.

\section{Generation and transduction of lentiviral shRNAs}

Lentiviral LeGO plasmids, shLUC and shRNA sequences were derived and previously validated in our prior studies ${ }^{17,40}$. shRor2-1 and shRor2-2 hairpin sequences correspond with shRor2-94 and -98 clones from the MISSION pLKO Lentiviral shRNA libraries (Sigma). Wnt pathway reporters (plasmids 7TGC \#24304, Addgene, Cambridge, MA, USA) were validated in mammary epithelial cells as described $^{17,40}$. Tumor cells $(500,000$ cells/well) were infected in 24-well plates at an MOI of 30 with 
lentivirus in growth media. Cells were briefly rinsed by phosphate-buffered saline after overnight infection, and then used in further experiments.

\section{Tumor organoids assays}

The organoids assays were performed in $2 \mathrm{mg} / \mathrm{ml}$ collagen gel. Rat-tail collagen I (\#354236; Corning) was diluted in 1x MEM at $2 \mathrm{mg} / \mathrm{ml}$ on ice and neutralized by $7.5 \%$ sodium bicarbonate solution. Eightwell chamber slides were coated with $5 \square \mu$ collagen and incubated at $37 \square{ }^{\circ} \mathrm{C}$ for 15 minutes. Aggregated organoids were washed in phosphate-buffered saline before being suspended in collagen at 50,000 cells $/ 40 \mu$ l volume. $40 \square \mu l$ of cell suspension was plated into each chamber and the chamber slides were incubated at $37 \square^{\circ} \mathrm{C}$ for 1 hour. After polymerization, collagen gel was overlaid with $500 \square \mu l$ of growth media. For RNA and protein extractions, collagen gel was dissociated in $2 \mathrm{mg} / \mathrm{ml}$ collagenase A solution. Organoids were then collected by a series of short centrifuges and washed prior to cell lysis.

\section{RNA isolation, sequencing, and analyses}

Total RNA was extracted and purified using RNeasy mini kit following the manufacturer's protocol (\#74104; Qiagen, Germantown, MD, USA). The BCM Genomic and RNA Profiling Core performed sample quality checks using the Nanodrop ND-1000 (ThermoFisher Scientific) and a Bioanalyzer Nano chip (Agilent Technologies, Santa Clara, CA, USA). RNA sequencing was also performed in the BCM Genomic and RNA Profiling Core. Differential gene expression analysis was performed using $\mathrm{R} /$ Bioconductor package edge $\mathrm{R}^{41}$. Gene ontology and pathway enrichment analysis were performed using DAVID Bioinformatics Resources 6.8.

\section{Quantitative real-time PCR}

Total RNA was reverse transcribed using high-capacity RNA-to-cDNA kit (\#4388950; Applied Biosystems, Foster City, CA, USA). Quantitative real-time PCR was performed by StepOnePlus RealTime PCR system using SYBR Green PCR master mix (Applied Biosystems). Primer sequences (Supplementary Table S1) were designed using the NCBI nucleotide BLAST. GAPDH was used as a 
reference gene for normalization, and relative gene expression fold changes were calculated as $2^{-\Delta \Delta \mathrm{Ct}}$

\section{Processing of tumors tissue and organoids cultures}

BrdU at $60 \square \mu \mathrm{g} / \mathrm{g}$ body weight was injected into the mice via intraperitoneal injection $2 \sqcap \mathrm{h}$ before tissue collection. Tissues were dissected and fixed in $4 \%$ paraformaldehyde overnight at $4 \square^{\circ} \mathrm{C}$ before processing to paraffin blocks. Organoid cultures were washed with phosphate-buffered saline and fixed in $4 \%$ paraformaldehyde for $5 \square$ min at room temperature before processing to paraffin blocks.

\section{Immunostaining}

Tissue and organoid sections were deparaffinized, rehydrated and subjected to sodium citrate or TrisEDTA antigen retrieval for $20 \square \mathrm{min}$. Sections were blocked at room temperature for $1 \mathrm{~h}$ by M.O.M. blocking solution (\#BMK-2202; Vector Laboratories, Burlingame, California, USA) containing 5\% BSA. Primary antibodies were applied overnight at $4 \square^{\circ} \mathrm{C}$. Antibodies and concentrations were: Ror2 (1:500; Developmental Studies Hybridoma Bank, lowa City, IA, USA), eGFP (1:1000; \#ab290; Abcam, Cambridge, MA, USA), RFP (1:1000; \#600-401-379; Rockland ,Pottstown, PA, USA), Integrin a5 (1:1000; \#ab150361, Abcam), Fibronectin 1 (1:2000; \#610077; BD Biosciences, San Jose, CA, USA), phospho-FAK (1:1000; \#44-626G; ThermoFisher Scientific), phospho-ERM (1:1000; CST, Danvers, MA, USA), cofilin (1:1000; \#5175S; CST), and phospho-cofilin (1:500; \#3313S; CST). The slides were washed three times in phosphate-buffered saline and incubated with Alexa Fluor 488conjugated or Alexa Fluor 594-conjugated goat anti-rabbit or anti-mouse IgG secondary antibodies in M.O.M. diluent containing $5 \%$ BSA in the dark for $1 \square \mathrm{h}$ at room temperature. The slides were washed three times in phosphate-buffered saline and counter stained with $1 \square \mu \mathrm{g} / \mathrm{ml}$ DAPI before being mounted with ProLong Diamond Antifade Mountant (P36961; ThermoFisher Scientific). Tyramide amplification was performed for Ror2 and phospho-FAK detection according to the manufacturer's instructions (\#NEL701A001KT; PerkinElmer, Waltham, MA, USA).

\section{Western blotting}


Protein samples were separated in NuPAGE 4-12\% Bis-Tris protein gels (\#NP0336BOX; ThermoFisher Scientific) and transferred to a PVDF membrane (\#LC2002; ThermoFisher Scientific). The blots were blocked with 5\% blocker (\#1706406; Bio-Rad, Hercules, CA, USA) in Tris-buffer saline containing $0.05 \%$ Tween-20. The blots were then incubated with primary antibodies overnight at $4 \square^{\circ} \mathrm{C}$ before incubating with secondary antibodies for $1 \mathrm{~h}$ at room temperature. The following antibodies were used in western blotting: Ror2 (1:1000, Developmental Studies Hybridoma Bank), Integrin a5 (1:1000; \#ab150361, Abcam), Fibronectin 1 (1:1000; \#610077; BD Biosciences), FAK (1:1000; \#3285; CST), phospho-FAK (1:1000; \#44-626G; ThermoFisher Scientific), cofilin (1:1000; \#5175S; CST), and GAPDH (1:2500; \#5174; CST).

\section{F-actin/G-actin assay}

Approximately $1 \times \square 10^{7}$ shLUC and shRor2 cells were harvested. F-actin and G-actin protein samples were collected according to the manufacturer's instructions (\#BK037, Cytoskeleton Inc., Denver, CO, USA). F-actin and G-actin were quantified by SDS-PAGE and western blot using actin antibody ((\#BK037, Cytoskeleton Inc.).

\section{Microscope image acquisition}

Confocal imaging and IncuCyte live imaging for this project was supported by the Integrated Microscopy Core at Baylor College of Medicine. The confocal images were acquired by a Nikon A1Rs confocal microscope with 20x (air), 40x (air), 40x (oil), and 100x (oil) objectives. The color channels were merged and converted to RBG images using Fiji 1.53c. The live imaging was performed on an IncuCyte S3 system. The image stacks were analyzed using Fiji 1.53c.

\section{Wound healing assay}

Cells (50,000 cells/well) were seeded into 2-well culture inserts (ibidi, Martinsried, Planegg, Germany) in 12-well plates. $200 \mu \mathrm{l}$ medium containing $1 \%$ FBS and indicated DMSO/inhibitors were added to each insert well. Inserts were removed on the following day, and $1 \mathrm{ml}$ of fresh medium containing $1 \%$ FBS and indicated DMSO/inhibitors were added into each well. Live imaging was performed using an 
IncuCyte S3 system and the images were analyzed using Wound Healing Tools in Fiji $1.53 c^{42}$. The time-lapse movies were generated by Fiji 1.53c.

\section{Statistical analysis and rigor and reproducibility}

Data are expressed as the mean $\square \pm \square$ s.d.; $n$ represents the number of biological replicates, unless specifically indicated otherwise in the figure legend. One-way ANOVA with Tukey's multiple comparison tests were performed on multi-group comparisons. Unpaired two-tailed Student's t-tests were performed on analyses involving two-group comparisons unless otherwise noted. Quantitative measurements were performed in ImageJ or GraphPad Prism 7. P<0.05 was considered statistically significant in all analyses. All experiments were reproduced across multiple $(\geq 3)$ biological replicates.

\section{Figure Legends}

Figure 1. Wnt/Ror2 regulates tumor cell-directed collagen remodeling and invasion independent of a complete EMT. (A, B) Representative trichrome staining of basal-like 2225L TP53null mammary tumors showing collagen abundance (blue) and tumor organization in shLUC (A) and shRor2 (B) tumor sections. Scale $50 \square \mu \mathrm{m}$. (C,D) Brightfield DIC images of shLUC and shRor2 2225L tumor organoids in Type I collagen. (scale $50 \mu \mathrm{m}$ ). Ror2-depleted organoids harbor increase invasive projections. (E) Quantitation of of the extent of migration in Ror2-depleted organoids within the basal-like 2225L and 2153L TP53null models. Representative of 3 independent experiments $\left(\mathrm{n}=20-30\right.$ organoids per group, $\left.{ }^{* * * *} \mathrm{p}<.0001,{ }^{* * *} \mathrm{p}<.001\right) .\left(\mathrm{F}-\mathrm{G}^{\prime}\right)$ Immunofluorescence of E-cadherin (green), vimentin (magenta), and nuclei (blue) in 2225L shLUC (F) and shRor2 (G,G') organoids, showing downregulated E-cadherin and altered distribution of vimentin upon Ror2 loss. G' shows downregulation of E-cadherin without upregulation of Vimentin. Scale $50 \square \mu \mathrm{m}$. (H) Western blot of 2225L shLUC and shRor2 organoids for Ror2 and E-cadherin, showing Ror2 depletion along with reduced E-cadherin expression levels in shRor2 organoids. (I) Western blot of 2225L shLUC and shRor2 organoids for vimentin. (J) Quantitative SYBR Green Real-time PCR measurement of mesenchymal markers (Vim, Zeb1, Snai1, and Snai2) in 2225L shLUC and shRor2 cells. Gene 
expression levels of the shRor2 group was normalized to the shLUC group and fold changes were graphed (ns: not significant; $n=3$ for each group).

Figure 2. Ror2 depletion promotes gene expression alterations related to cell-ECM interactions, integrin signaling, and cytoskeleton reorganization in tumor organoids. (A) Heatmap display of significantly differentially expressed genes $(p<0.01$ and fold change $>1.2)$ in 2225L shLUC and shRor2 organoids by RNA sequencing. Fold changes are represented by two-way gradients to blue (downregulation) and orange (upregulation). (B) Gene ontology analysis demonstrating the enrichment of gene expression in particular biological processes in 2225L shRor2 organoids. (C) Heatmap display of significantly differentially expressed genes $(p<0.01$ and fold change $>1.2)$ in $2153 \mathrm{~L}$ shLUC and shRor2 organoids by RNA sequencing. Fold changes are represented by two-way gradients to blue (downregulation) and orange (upregulation). (D) Gene ontology analysis demonstrating the enrichment of gene expression in particular biological processes in 2153L shRor2 organoids. (E) Heatmap display of highlighted genes in 2225L shLUC and shRor2 organoids related to cell-ECM interaction, integrin signaling, and cytoskeleton reorganization. Fold changes are represented by two-way gradients to blue (downregulation) and orange (upregulation). (F) Quantitative Real-time PCR measurement of highlighted genes in 2225L shLUC and shRor2 cells. Gene expression levels of the shRor2 group was normalized to the shLUC group and fold changes were graphed $\left({ }^{* * *}: p<0.0001 ;{ }^{* *}: p<0.001 ; n=3\right.$ for each group).

Figure 3. Integrin a5 and its ligand, fibronectin 1, are upregulated and spatially restructured upon Ror2 depletion. (A) Western blot of 2225L shLUC and shRor2 organoids for Integrin a5 heavy chain and light chain, showing increased integrin a5 expression levels in Ror2 depleted organoids. (B) Immunofluorescence of integrin a5 (green), E-cadherin (magenta), and nuclei (blue) in 2225L shLUC organoids. Scale $50 \square \mu \mathrm{m}$ (C) Immunofluorescence of integrin a5 (green), E-cadherin (magenta), and nuclei (blue) in 2225L shRor2 organoids, showing upregulated integrin a5 upon Ror2 loss, especially in the invasive projections. Scale $50 \square \mu \mathrm{m}$. (D) Immunofluorescence of integrin a5 (green), pan-Keratin (magenta), and nuclei (blue) in 2225L shLUC primary tumor sections, showing 
topographic distribution of integrin a5 in control tumors. Scale $100 \square \mu \mathrm{m}$. (D') integrin a5 staining in the periphery of shLUC tumor. (D") integrin a5 staining in the body of shLUC tumors. Scale $100 \square \mu \mathrm{m}$. (E) Representative immunofluorescence of integrin a5 (green), pan-Keratin (magenta), and nuclei (blue) in 2225L shRor2 primary tumor sections, showing enhanced integrin a5 expression within and around tumor cells in shRor2 tumors. Scale $100 \square \mu \mathrm{m}$. (E') integrin a5 staining in the periphery of shRor2 tumors. (E") integrin a5 staining in the body of shRor2 tumors. Scale $100 \square \mu \mathrm{m}$. (F) Western blot of 2225L shLUC and shRor2 organoids for fibronectin 1, showing increased fibronectin 1 expression levels in Ror2 depleted organoids. (G) Immunofluorescence of fibronectin 1 (green), pan-Keratin (magenta), and nuclei (blue) in 2225L shLUC organoids. Scale $50 \square \mu \mathrm{m}(\mathrm{H})$ Immunofluorescence of fibronectin 1 (green), pan-Keratin (magenta), and nuclei (blue) in 2225L shRor2 organoids, showing increased fibronectin 1 deposition upon Ror2 loss, especially near the invasive projections at the tumor cell-matrix interface. Scale $50 \square \mu \mathrm{m}$. (I) Immunofluorescence of fibronectin 1 (green), RFP (magenta), and nuclei (blue) in 2225L shLUC primary tumor sections, showing deposition of fibronectin 1 in control tumors. Scale $100 \square \mu \mathrm{m}$. (I') fibronectin 1 staining in the periphery of shLUC tumor. (I") fibronectin 1 staining in the body of shLUC tumor. Scale $100 \square \mu \mathrm{m}$. (J) Immunofluorescence of fibronectin 1 (green), RFP (magenta), and nuclei (blue) in 2225L shRor2 primary tumor sections, showing increased fibronectin 1 deposition around tumor cells in shRor2 tumors. Scale $100 \square \mu \mathrm{m} .\left(\mathrm{J}^{\prime}\right)$ fibronectin 1 staining in the periphery of shRor2 tumor. $\left(\mathrm{J}^{\prime \prime}\right)$ fibronectin 1 staining in the body of shRor2 tumor. Scale $100 \square \mu \mathrm{m}$.

Figure 4. Focal adhesions and actin cytoskeleton dynamics are regulated by Ror2 (A) Western blot of 2225L shLUC and shRor2 organoids for phosphorylated FAK and total FAK showing increased FAK phosphorylation in Ror2 depleted organoids. (B,C) Immunofluorescence of p-FAK (yellow), Factin (magenta), and nuclei (blue) in 2225L organoids, showing changes in p-FAK and F-actin (measured by LifeAct-eGFP) upon Ror2 loss (C). Scale 100 $\square$ mm. (D) Immunofluorescence of p-FAK (yellow), pan-Keratin (magenta), and nuclei (blue) in 2225L shLUC primary tumor sections, showing topographic distribution of p-FAK in control tumors. Scale $100 \square \mu m$. (D') p-FAK staining in the 
periphery of shLUC tumor. (D") p-FAK staining in the body of shLUC tumor. Scale 100 $\square \mu m$. (E) Immunofluorescence of p-FAK (yellow), pan-Keratin (magenta), and nuclei (blue) in 2225L shRor2 primary tumor sections, showing topographic alterations in p-FAK distribution upon Ror2 loss. Scale $100 \square \mu \mathrm{m}$. (D') p-FAK staining in the periphery of shRor2 tumor. (E") p-FAK staining in the body of shRor2 tumor. Scale $100 \square \mu \mathrm{m}$. (F) F-actin/G-actin ratio assay of $2225 \mathrm{~L}$ shLUC and shRor2 cells showing increased F-actin content in Ror2 depleted cells. (G) Quantification of the F-actin/G-actin ratio in $2225 \mathrm{~L}$ shLUC and shRor2 cells ( ${ }^{\star * *}: \mathrm{p}<0.0001 ; n=3$ biological replicates). $(\mathrm{H})$ Western blot of 2225L shLUC and shRor2 organoids for cofilin showing reduced cofilin expression in Ror2 depleted organoids. (I) Immunofluorescence of cofilin (yellow), pan-Keratin (magenta), and nuclei (blue) in 2225L shLUC primary tumor sections, showing expression and localization of cofilin in control tumors. Scale $100 \square \mu \mathrm{m}$. (I') Cofilin staining in the periphery of shLUC tumor. (J) Immunofluorescence of cofilin (yellow), pan-Keratin (magenta), and nuclei (blue) in 2225L shRor2 primary tumor sections, showing decreased total cofilin expression upon Ror2 depletion. Scale $100 \square \mu \mathrm{m}$. (J') Cofilin staining in the periphery of shRor2 tumor. (K) Immunofluorescence of p-cofilin (yellow), pan-Keratin (magenta), and nuclei (blue) in 2225L shLUC primary tumor sections, showing expression and localization of cofilin in control tumors. Scale 100 $\square$ m. (L) Immunofluorescence of p-cofilin (yellow), pan-Keratin (magenta), and nuclei (blue) in 2225L shRor2 primary tumor sections, showing increased p-cofilin upon Ror2 depletion. Scale 100 $\square$ m. (M) Immunofluorescence of p-ERM (yellow), pan-Keratin (magenta), and nuclei (blue) in 2225L shLUC primary tumor sections, showing expression and localization of cofilin in control tumors. Scale $100 \square \mu \mathrm{m}$. (M,N) Immunofluorescence of p-ERM (yellow), pan-Keratin (magenta), and nuclei (blue) in $2225 \mathrm{~L}$ shRor2 primary tumor sections, showing increased and redistributed p-ERM upon Ror2 depletion. Scale 100 $\square$ m.

Figure 5. Inhibition of integrin a5 or FAK compromised breast cancer cell migration and invasion induced by Ror2 depletion. (A) Western blot of $2225 \mathrm{~L}$ shLUC and shRor2 cells for

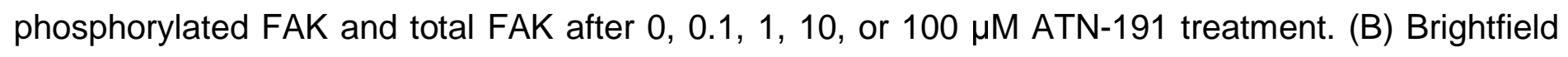
images of wound healing assay on $2225 \mathrm{~L}$ shLUC and shRor2 cells with or without integrin a5 
inhibition (10 $\mu \mathrm{M}$ ATN-191) showing cell migration within 16 hours. (C) Quantification of the ratio of remaining wound area after 16 hours to the initial wound area with or without integrin a5 inhibition. (D) Western blot of 2225L shLUC and shRor2 organoids for phosphorylated FAK and total FAK after 10 $\mu \mathrm{M}$ ATN-191 treatment. (E) Western blot of $2225 \mathrm{~L}$ shLUC and shRor2 cells for phosphorylated FAK and total FAK after 0, 10, 50, 100, or 200 nM PF-562271 Besylate treatment. (F) Brightfield images of wound healing assay on 2225L shLUC and shRor2 cells with or without FAK inhibition (200 nM PF562271 Besylate) showing cell migration within 16 hours. (G) Quantification of the ratio of remaining wound area after 16 hours to the initial wound area with or without FAK inhibition. $(\mathrm{H})$ Western blot of 2225L shLUC and shRor2 organoids for phosphorylated FAK and total FAK after 200 nM PF-562271 Besylate treatment. (I) Brightfield DIC images showing compromised invasion of shRor2 organoids into the surrounding matrix after integrin a5 or FAK inhibition. Scale $100 \square \mu \mathrm{m}$. (J) Quantitation of cellular projections emanating into the surrounding matrix in shRor2 vs. shLUC organoids ( $p$-value noted in the panel; ns: not significant; $n=23,9,16,22,10$, and 16 organoids for shLUC + vehicle, shLUC + integrin a5 inhibition, shLUC + FAK inhibition, shRor2 + vehicle, shRor2 + integrin a5 inhibition, and shRor2 + FAK inhibition group, respectively).

\section{Acknowledgements and Funding}

We want to thank Jeffrey Rosen Ph.D. for his dedication to breast cancer research, collaboration, and mentorship, as well as the abundance of scientific discussions surrounding the current work. Kevin Roarty is supported by NCl 5K22CA207463, Susan G. Komen CCR18548284, a Dan L. Duncan Comprehensive Cancer Center Faculty Scholar Award, L.E Gordon Cancer Research Fund, Caroline Wiess Law Fund for Research in Molecular Medicine, and a Breast SPORE Career Enhancement Award 2P50CA186784-06 (PI: Ellis). H.S and N.Z. were additionally supported by CA1082708311. We also thank Fengju Chen for bioinformatics support within the Biostatistics and Cancer Bioinformatics Division of the DLDCCC. This project was also supported by the Genomic and RNA Profiling Core at Baylor College of Medicine and the Cytometry and Cell Sorting Core at Baylor 
the NIH (P30 CA125123 and S10 RR024574) and the expert assistance of Joel M. Sederstrom.

\section{References}

1. Chiang, A.C. \& Massague, J. Molecular basis of metastasis. N Engl J Med 359, 2814-2823 (2008).

2. Friedl, P. Prespecification and plasticity: shifting mechanisms of cell migration. Curr Opin Cell Biol 16, 14-23 (2004).

3. Friedl, P. \& Alexander, S. Cancer invasion and the microenvironment: plasticity and reciprocity. Cell 147, 992-1009 (2011).

4. Kauppila, S., Stenback, F., Risteli, J., Jukkola, A. \& Risteli, L. Aberrant type I and type III collagen gene expression in human breast cancer in vivo. J Pathol 186, 262-268 (1998).

5. Provenzano, P.P., et al. Collagen density promotes mammary tumor initiation and progression. $B M C$ Med 6, 11 (2008).

6. Levental, K.R., et al. Matrix crosslinking forces tumor progression by enhancing integrin signaling. Cell 139, 891-906 (2009).

7. Rottenberg, S., et al. High sensitivity of BRCA1-deficient mammary tumors to the PARP inhibitor AZD2281 alone and in combination with platinum drugs. Proc Natl Acad Sci U S A 105, 17079-17084 (2008).

8. Erler, J.T., et al. Hypoxia-induced lysyl oxidase is a critical mediator of bone marrow cell recruitment to form the premetastatic niche. Cancer Cell 15, 35-44 (2009).

9. McLean, G.W., et al. The role of focal-adhesion kinase in cancer - a new therapeutic opportunity. Nat Rev Cancer 5, 505-515 (2005).

10. Hamidi, H. \& Ivaska, J. Every step of the way: integrins in cancer progression and metastasis. Nat Rev Cancer 18, 533-548 (2018).

11. Lintz, M., Munoz, A. \& Reinhart-King, C.A. The Mechanics of Single Cell and Collective Migration of Tumor Cells. J Biomech Eng 139(2017).

12. Friedl, P. \& Gilmour, D. Collective cell migration in morphogenesis, regeneration and cancer. Nat Rev Mol Cell Biol 10, 445-457 (2009).

13. Franz, C.M., Jones, G.E. \& Ridley, A.J. Cell migration in development and disease. Dev Cell 2, 153-158 (2002).

14. van Amerongen, R. \& Nusse, R. Towards an integrated view of Wnt signaling in development. Development 136, 3205-3214 (2009).

15. Angers, S. \& Moon, R.T. Proximal events in Wnt signal transduction. Nat Rev Mol Cell Biol 10, 468-477 (2009).

16. Marusyk, A., Janiszewska, M. \& Polyak, K. Intratumor Heterogeneity: The Rosetta Stone of Therapy Resistance. Cancer Cell 37, 471-484 (2020).

17. Roarty, K., Pfefferle, A.D., Creighton, C.J., Perou, C.M. \& Rosen, J.M. Ror2-mediated alternative Wnt signaling regulates cell fate and adhesion during mammary tumor progression. Oncogene (2017).

18. Cox, T.R. \& Erler, J.T. Remodeling and homeostasis of the extracellular matrix: implications for fibrotic diseases and cancer. Dis Model Mech 4, 165-178 (2011).

19. Kai, F., Drain, A.P. \& Weaver, V.M. The Extracellular Matrix Modulates the Metastatic Journey. Dev Cell 49, 332-346 (2019). 
20. Pickup, M.W., Mouw, J.K. \& Weaver, V.M. The extracellular matrix modulates the hallmarks of cancer. EMBO Rep 15, 1243-1253 (2014).

21. Jerry, D.J., et al. A mammary-specific model demonstrates the role of the p53 tumor suppressor gene in tumor development. Oncogene 19, 1052-1058 (2000).

22. Hynes, R.O. Integrins: versatility, modulation, and signaling in cell adhesion. Cell 69, 11-25 (1992).

23. Pankov, R., et al. Integrin dynamics and matrix assembly: tensin-dependent translocation of alpha(5)beta(1) integrins promotes early fibronectin fibrillogenesis. J Cell Biol 148, 1075-1090 (2000).

24. Wu, C., Bauer, J.S., Juliano, R.L. \& McDonald, J.A. The alpha 5 beta 1 integrin fibronectin receptor, but not the alpha 5 cytoplasmic domain, functions in an early and essential step in fibronectin matrix assembly. J Biol Chem 268, 21883-21888 (1993).

25. Joshi, R., Goihberg, E., Ren, W., Pilichowska, M. \& Mathew, P. Proteolytic fragments of fibronectin function as matrikines driving the chemotactic affinity of prostate cancer cells to human bone marrow mesenchymal stromal cells via the alpha5beta1 integrin. Cell Adh Migr 11, 305-315 (2017).

26. Gopal, S., et al. Fibronectin-guided migration of carcinoma collectives. Nat Commun 8, 14105 (2017).

27. Missirlis, D., Haraszti, T., Kessler, H. \& Spatz, J.P. Fibronectin promotes directional persistence in fibroblast migration through interactions with both its cell-binding and heparin-binding domains. Sci Rep 7, 3711 (2017).

28. Dohn, M.R., Mundell, N.A., Sawyer, L.M., Dunlap, J.A. \& Jessen, J.R. Planar cell polarity proteins differentially regulate extracellular matrix organization and assembly during zebrafish gastrulation. Dev Biol 383, 39-51 (2013).

29. Parisi, L., et al. A glance on the role of fibronectin in controlling cell response at biomaterial interface. Jpn Dent Sci Rev 56, 50-55 (2020).

30. Kanellos, G. \& Frame, M.C. Cellular functions of the ADF/cofilin family at a glance. J Cell Sci 129, 32113218 (2016).

31. Vega, M.E. \& Schwarzbauer, J.E. Collaboration of fibronectin matrix with other extracellular signals in morphogenesis and differentiation. Curr Opin Cell Biol 42, 1-6 (2016).

32. Williams, E.D., Gao, D., Redfern, A. \& Thompson, E.W. Controversies around epithelial-mesenchymal plasticity in cancer metastasis. Nat Rev Cancer 19, 716-732 (2019).

33. Carvalho, J.R., et al. Non-canonical Wnt signaling regulates junctional mechanocoupling during angiogenic collective cell migration. Elife 8(2019).

34. le Duc, Q., et al. Vinculin potentiates E-cadherin mechanosensing and is recruited to actin-anchored sites with in adherens junctions in a myosin II-dependent manner. J Cell Biol 189, 1107-1115 (2010).

35. Spill, F., Reynolds, D.S., Kamm, R.D. \& Zaman, M.H. Impact of the physical microenvironment on tumor progression and metastasis. Curr Opin Biotechnol 40, 41-48 (2016).

36. Sottile, J. \& Hocking, D.C. Fibronectin polymerization regulates the composition and stability of extracellular matrix fibrils and cell-matrix adhesions. Mol Biol Cell 13, 3546-3559 (2002).

37. Kadler, K.E., Hill, A. \& Canty-Laird, E.G. Collagen fibrillogenesis: fibronectin, integrins, and minor collagens as organizers and nucleators. Curr Opin Cell Biol 20, 495-501 (2008).

38. Erdogan, B., et al. Cancer-associated fibroblasts promote directional cancer cell migration by aligning fibronectin. J Cell Biol 216, 3799-3816 (2017).

39. Dzamba, B.J., Jakab, K.R., Marsden, M., Schwartz, M.A. \& DeSimone, D.W. Cadherin adhesion, tissue tension, and noncanonical Wnt signaling regulate fibronectin matrix organization. Dev Cell 16, 421-432 (2009).

40. Roarty, K., Shore, A.N., Creighton, C.J. \& Rosen, J.M. Ror2 regulates branching, differentiation, and actin-cytoskeletal dynamics within the mammary epithelium. J Cell Biol 208, 351-366 (2015).

41. Huber, W., et al. Orchestrating high-throughput genomic analysis with Bioconductor. Nat Methods 12, 115-121 (2015).

42. Suarez-Arnedo, A., et al. An image J plugin for the high throughput image analysis of in vitro scratch wound healing assays. PLoS One 15, e0232565 (2020). 
bioRxiv preprint doi: https://doi.org/10.1101/2021.05.15.444295; this version posted May 16, 2021. The copyright holder for this preprint (which was not certified by peer review) is the author/funder, who has granted bioRxiv a license to display the preprint in perpetuity. It is made available under aCC-BY-NC-ND 4.0 International license. 


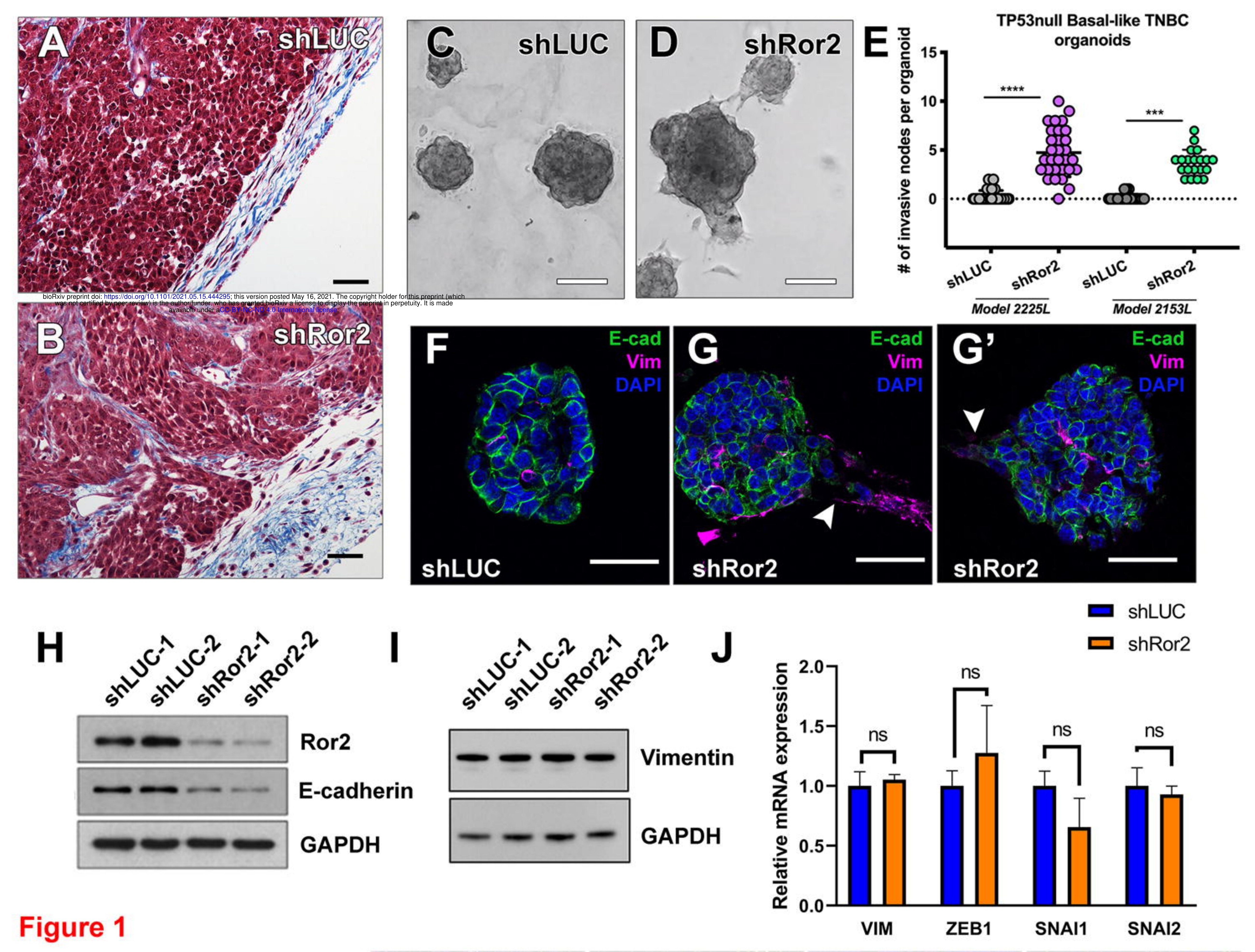


2225L TP53null organoids shRor2 vs. shLUC
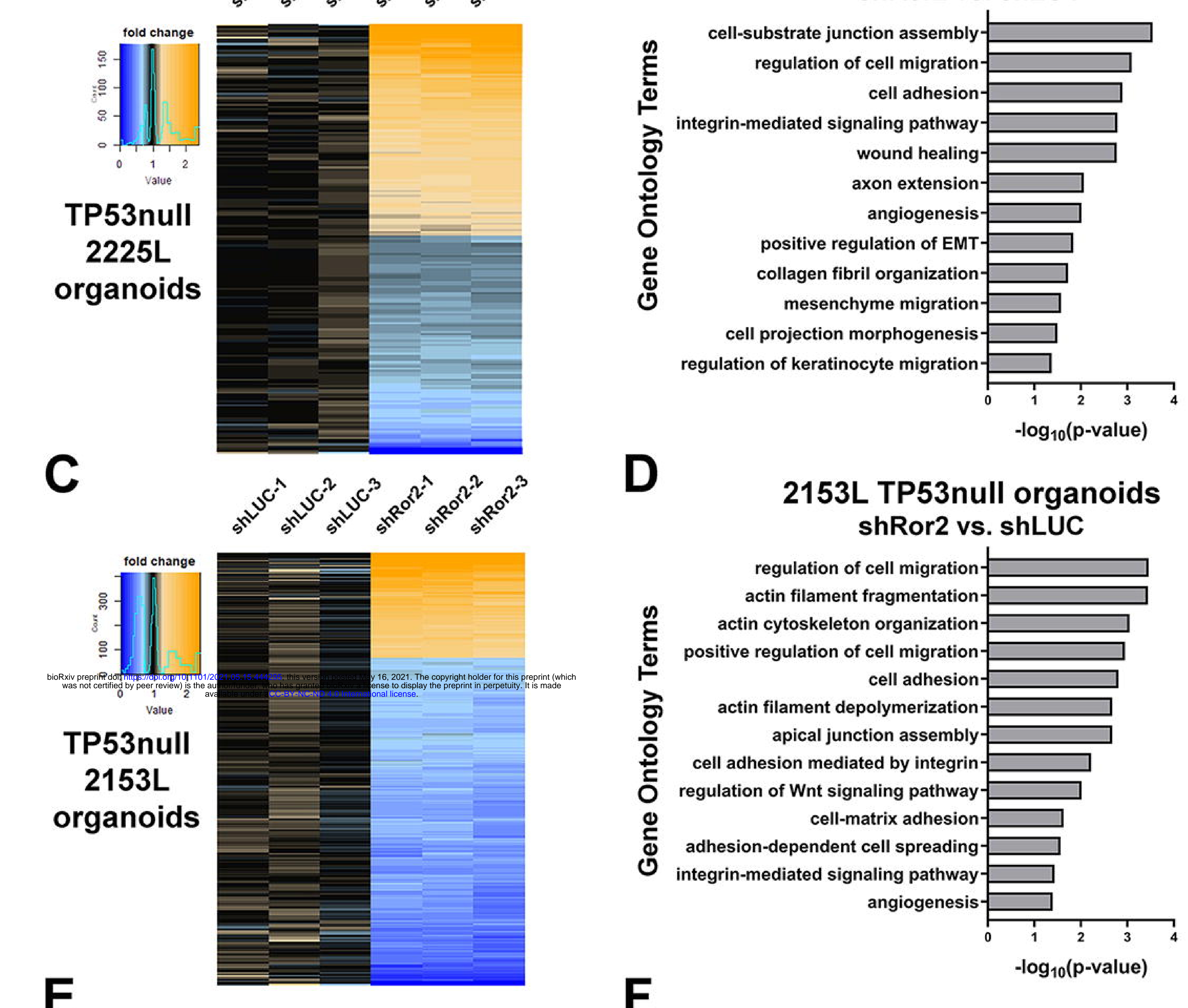

TP53null

2225L organoids

롱

\section{L TP53null organoids shRor2 vs. shLUC}

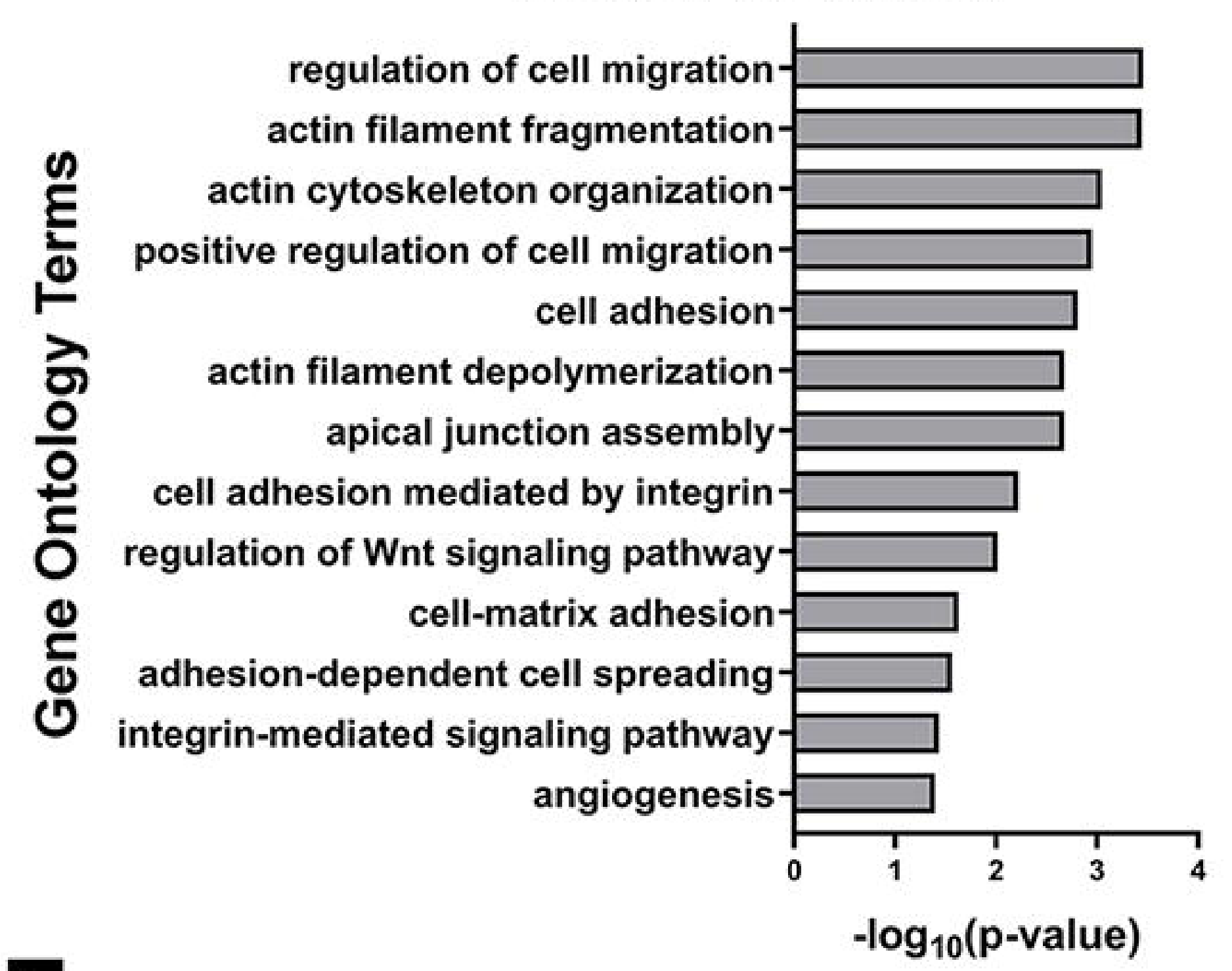

$\mathbf{F}$

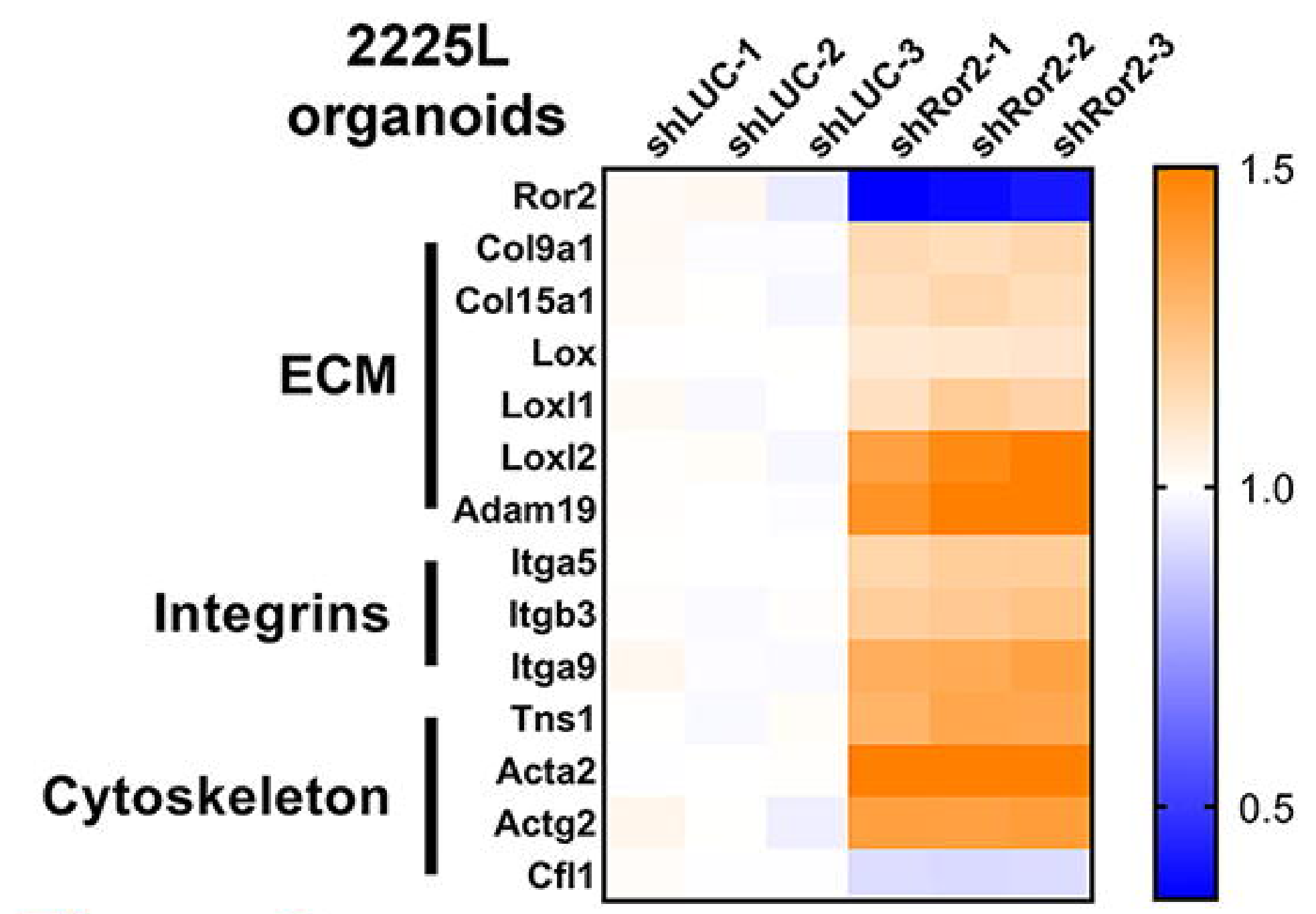

\section{Figure 2}

\section{TP53null 2225L tumor cells}

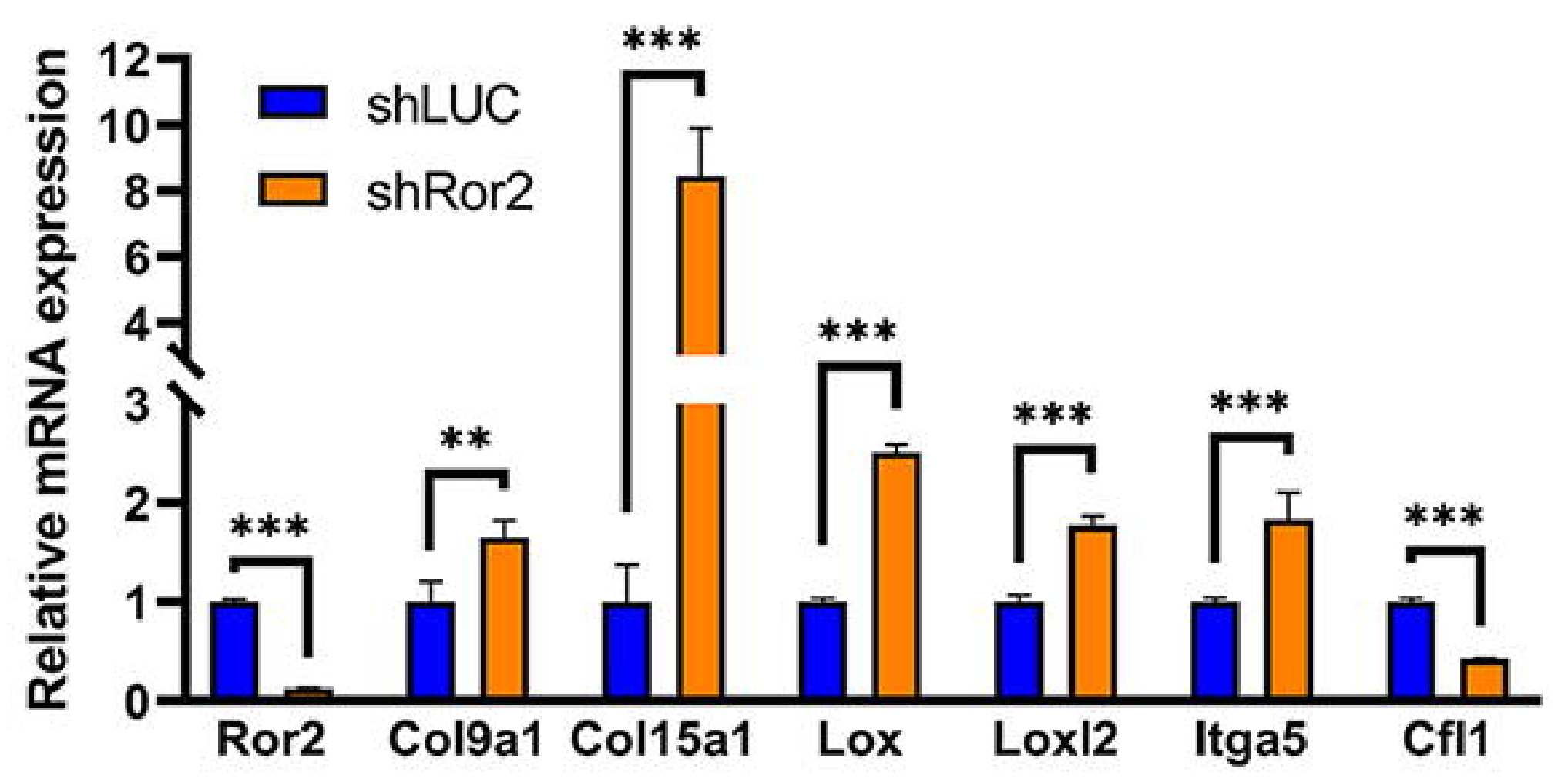




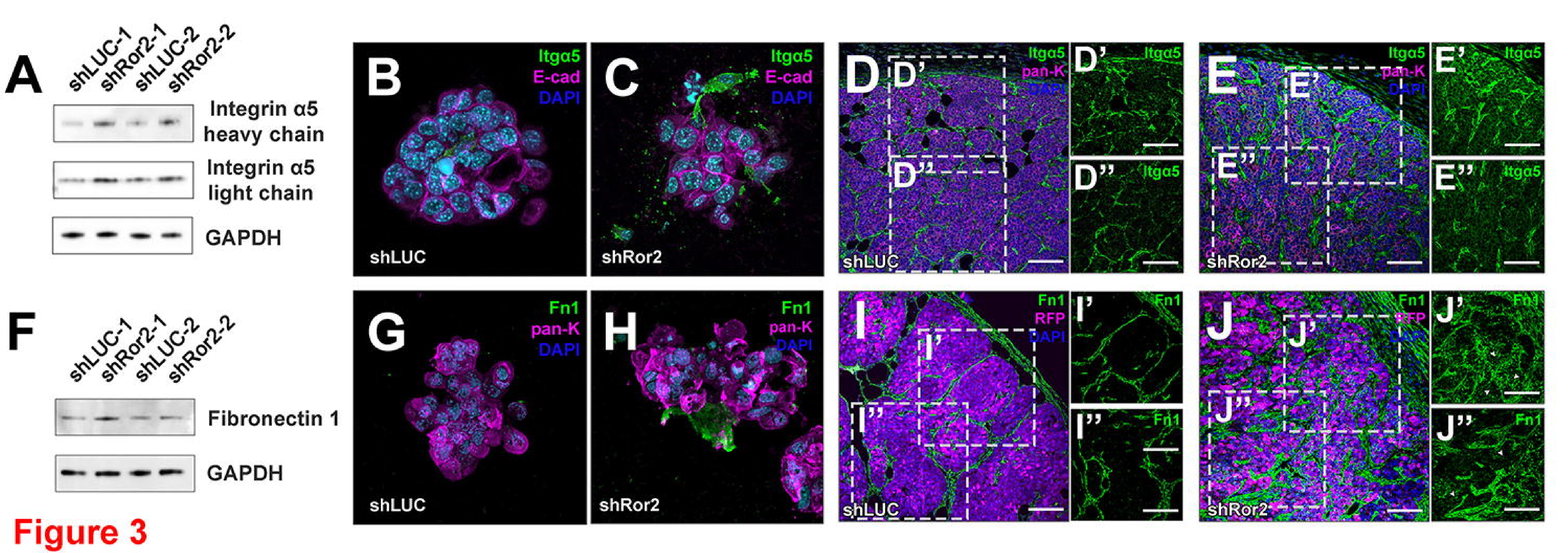




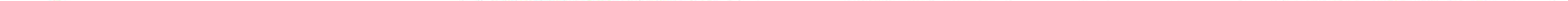


